\title{
Congenital Lactobacillus Blood Stream Infection in Extremely Preterm Twins
}

\author{
Peter Korček $^{1,2} \cdot$ Zbyněk Straňák $^{1,2} \cdot$ Václav Čunát ${ }^{1}$
}

Received: 4 December 2015 / Accepted: 26 April 2016/Published online: 13 May 2016

(C) Dr. K C Chaudhuri Foundation 2016

To the Editor: Lactobacillus spp. blood stream infection is extremely rare in children and is predominantly described in the adult population [1]. Only a few reports exist regarding neonatal Lactobacillus infection.

A 31-y-old woman with preterm dichorionic diamniotic twins was admitted due to the rupture of membranes. Tocolytics, antenatal corticosteroids and antibiotics were administered in view of chorioamnionitis (patient history, elevated C-reactive protein). Inflammatory markers escalated despite treatment and failed tocolysis led to cesarean section at $24+5$ wk gestational age. Both infants suffered from severe respiratory distress syndrome and required prolonged ventilatory support. The infants were started on Penicillin and Gentamicin, however, no serious signs of sepsis were observed and the blood sample at admission revealed no significant elevation of inflammatory markers. Nevertheless, blood cultures were positive for Lactobacillus spp. The infants were treated successfully with antibiotics for seven days and posttreatment blood cultures were negative. The infants' outcome was favourable without any serious adverse events.

The main risk factors for Lactobacillus infection appear to be immunodeficiency, severe underlying disease, invasive lines or prolonged ineffective antibiotic treatment [1]. Although congenital Lactobacillus infection is uncommon

Zbyněk Straňák

z.stranak@seznam.cz

1 Fetal Medicine Center, Institute for the Care of Mother and Child, Podolské nábreží, 157 Prague, Czech Republic

2 Third Faculty of Medicine, Charles University, Prague, Czech Republic and has been reported previously, this complication has not been yet described in extremely preterm twins.

In our case, the twins' blood cultures at admission were positive for Lactobacillus spp. Interestingly, the inflammatory response in infants was minimal including the twin $\mathrm{A}$, where histological chorioamnionitis was confirmed. The infection transmission remains unclear as we were unable to cultivate Lactobacillus spp. from any other site (neonatal, placental and cervicovaginal swabs). However, the contamination was considered unlikely as blood cultures were obtained by two skilled neonatologists in a tertiary center.

Cultured from blood stream using sterile technique, Lactobacillus should be recognized as a serious pathogen regardless of mild clinical and laboratory findings, especially in extremely preterm infants $[2,3]$. We can speculate about different outcome in our patients if the optimal antibiotic treatment was not given.

\section{Compliance with Ethical Standards}

Conflict of Interest None.

Source of Funding None.

\section{References}

1. Salminen MK, Rautelin H, Tynkkynen S, et al. Lactobacillus bacteremia, clinical significance, and patient outcome, with special focus on probiotic L. rhamnosus GG. Clin Infect Dis. 2004. doi:10.1086/ 380455.

2. Thompson C, McCarter YS, Krause PJ, Herson VC. Lactobacillus acidophilus sepsis in a neonate. J Perinatol. 2001;21:258-60.

3. Sadowska-Krawczenko I, Paprzycka M, Korbal P, et al. Lactobacillus rhamnosus GG suspected infection in a newborn with intrauterine growth restriction. Benef Microbes. 2014;5:397-402. 